\title{
To know the correct diagnosis and the effect of COVIDI9 on the various organs of the body, it is mandatory to make autopsy
}

\section{Letter to editor}

It's quite known nowadays about the outbreak of the Corona virus that has broken through our world and what consequences it has brought to us and the God knows what it might bring in the near future. Precautions have been taken by the global community: WHO, official authorities, Government, health institutions, medical staff, in all fields and by the public alike. I'm sure it's a great responsibility to be taken considering all the non-stop efforts of medical experts and physicians in the attempts to fight and stop the disease invasion and transmission, and treat the patients. Yet, I have noticed that in spite of all the efforts, no one knows the specific symptoms and the exact cause of the patients death(including WHO).There is currently a lack of pathologic data on the novel coronavirus (severe acute respiratory syndrome coronavirus 2) pneumonia, or coronavirus disease 2019 (COVID-19)from an autopsy or biopsy. ${ }^{1}$ Also, none of the forensic pathologists have offered to perform an autopsy to one of the corpses died by corona virus only, as the main unique next step for spot medical diagnosis. I'm quite aware of the fright of the doctors from catching the virus if an attempt to do so has commenced, but one must say it's a must-do- step, as there is no pathologic studies have been conducted on the basis of autopsies or biopsies. Some of the reasons for the lack of autopsies and biopsies include suddenness of the outbreak, vast patient, volume in hospitals, shortage of health care personnel and high rate of transmission, which makes invasive diagnostic procedures less of a clinical priority. ${ }^{1}$

In my long work and experience in the field of the forensic medicine, I see an urge for someone to move to the next serious step- performing an autopsy - if you wish to enrich yourselves and the world with new knowledge about this virus for participation and solution, and take the diagnostic characteristics according to WHO report, ${ }^{2}$ which supports my suggestion, is the following which have been published, the experts stated:

Since the start of the COVID-19 outbreak, there have been extensive attempts to better understand the virus and the disease in China. It is remarkable how much knowledge about a new virus has been gained in such a short time. However, as with all new diseases, and only 7 weeks after this outbreak began, key knowledge gaps remain. The key is unknown in a number of areas including the source of infection, pathogenesis and virulence of the virus, transmissibility, risk factors for infection and disease progression, surveillance, diagnostics, clinical management of severe and critically ill patients, and the effectiveness of prevention and control measures. The timely filling of these knowledge gaps is imperative to enhance control strategies. ${ }^{2}$ And they added: typical signs and symptoms include: fever, dry cough, fatigue, sputum production, shortness of breath, sore throat, headache, myalgia or arthralgia, chills, nausea or vomiting, nasal congestion, diarrhea, hemoptysis and conjunctival congestion, ${ }^{2}$ (with different static percentages). Look to the contradiction in symptoms: dry and productive (sputum) cough, how could this happen? Also they added: symptoms of COVID-19 are non-specific and the disease
Volume 8 Issue 3 - 2020

\author{
Othman Alfleesy \\ Department of Forensic Medicine and Pathology, Faculty of \\ Medicine and Health Sciences, Aden University, South Yemen \\ Correspondence: Othman Alfleesy, Associate Professor \\ of Forensic Medicine, Department of Forensic Medicine and \\ Pathology, Faculty of Medicine and Health Sciences, Aden \\ University, Aden city, South Yemen, \\ Email alfleesy200I@yahoo.com
}

Received: May 09, 2020 | Published: May 29, 2020

presentation can range from no symptoms(asymptomatic) to severe pneumonia and death. This is not true and nonsense, because we still don't know its main symptoms and clinical features, but definitely it has its characteristics. Even WHO experts deliver wrong information, simply, because the virus is unique, and human being is unique too. So, the symptom must be the same, but may differ in its severity according to status of the patient from different aspects. So, from available data, and in the absence of correct and trust results, it is not possible to determine the exact cause of death, and to define the symptoms belong to this virus or others according to WHO report. ${ }^{2}$ So, I feel like, it's my obligation to offer you my services as a forensic medicine specialist and to come forward and help the other doctors in their fierce battle against our new enemy. Of course, I am afraid of this fatal disease like you, but I have strict personal precautions and conditions to fulfill this task. But of course none of this can happen without intensely strict safety precautions as you're quite aware of the disease and how it works. If my letter finds a response from any health organization in any country, I am ready to fulfill this task after acceptance of my available precautions and conditions.

\section{Acknowledgments}

None.

\section{Conflicts of interest}

There is no conflict of interest.

\section{References}

1. Sufang Tian, Weidong Hu, Li Niu, et al., Pulmonary Pathology of Early-Phase 2019 Novel Coronavirus (COVID-19), Pneumonia in Two Patients With Lung Cancer. Journal of thoracic oncology. 2020;15(5):700-704.

2. Report of the WHO-China Joint Missionon Coronavirus Disease 2019 (COVID-19). 2020. 